\title{
Bronchopulmonary Dysplasia: Can We Agree on a Definition?
}

\author{
Eduardo Bancalari, MD ${ }^{1}$ Deepak Jain, MD ${ }^{1}$ \\ 1 Division of Neonatology, Department of Pediatrics, \\ University of Miami Medical School, Miami, Florida \\ Am J Perinatol 2018;35:537-540.
}

\begin{abstract}
Address for correspondence Eduardo Bancalari, MD, Division of Neonatology, Department of Pediatrics, University of Miami Medical School, Miami, 1611 Northwest, 12th Avenue, Miami, FL 33136 (e-mail: ebancalari@med.miami.edu).
\end{abstract}

\begin{abstract}
Keywords

- premature

- chronic lung disease

- definition

The advances in obstetric and neonatal care over the last half century have resulted in changes in pathophysiology and clinical presentation of bronchopulmonary dysplasia (BPD). In contrast to the original description of BPD by Northway et al as a severe lung injury in relatively mature preterm infants, the most common form of BPD currently is characterized by chronic respiratory insufficiency in extremely preterm infants. This evolution in the presentation of BPD, along with changes in respiratory support strategies such as increased use of nasal cannula oxygen, has presented a unique challenge to find a definition that describes the severity of lung damage and predict the long-term respiratory outcomes with some accuracy.

The limitations of current definitions of BPD include inconsistent correlation with longterm respiratory outcomes, inability to classify infants dying from severe respiratory failure prior to 36 weeks' postmenstrual age, and potential inappropriate categorization of infants on nasal cannula oxygen or with extrapulmonary causes of respiratory failure. In the long term, the aim for a new definition of BPD is to develop a classification based on the pathophysiology and objective lung function evaluation providing a more accurate assessment for individual patients. Until then, a consensus definition that encompasses current clinical practices, provides reasonable prediction of later respiratory outcomes, and is relatively simple to use should be achieved.
\end{abstract}

Since its first description by Northway et al 50 years ago, ${ }^{1}$ bronchopulmonary dysplasia (BPD) continues to be the most significant respiratory morbidity affecting premature infants. Significant advances in obstetrics and neonatal medicine over the last half century have resulted in an evolution in the pathophysiology and clinical picture of BPD. In contrast to Northway et al's description of BPD as the end result of severe lung injury due to high ventilator pressures and oxygen concentrations in relatively mature preterm infants, the most common form of BPD today is the end result of a complex interplay between abnormal lung development and injury/ repair in extremely preterm infants. This has presented a unique challenge for neonatologists to find a definition that describes the severity of the lung damage and at the same time can predict with some accuracy long-term respiratory outcome for a disease that is continuously evolving.

\section{Evolution of the Definition of Bronchopulmonary Dysplasia}

To overcome the problem of changes in pathogenesis over time, most diagnostic criteria have used treatment variables such as oxygen concentration and positive pressure at different time points during the clinical course. With improving survival of extremely immature infants and changes in care practices, these diagnostic criteria have been modified multiple times. ${ }^{2,3}$ In a major review in 2001, Jobe and Bancalari proposed severity-based diagnostic criteria for BPD that included oxygen need for 28 days and an assessment of respiratory support at 36 weeks' postmenstrual age (PMA; - Table 1). ${ }^{4}$ This definition has been used inconsistently with various modified versions either to more accurately assess the need for oxygen by room air challenge test at the
Copyright (c) 2018 by Thieme Medical Publishers, Inc., 333 Seventh Avenue, New York, NY 10001, USA. Tel: +1(212) 584-4662.
DOI https://doi.org/ 10.1055/s-0038-1637761. ISSN 0735-1631. 
Table 1 NIH workshop definition of BPD

\begin{tabular}{|l|l|l|}
\hline Gestational age & $<32$ wk & $\geq 32$ wk \\
\hline $\begin{array}{l}\text { Time point of } \\
\text { assessment }\end{array}$ & $\begin{array}{l}\text { 36 wk' PMA or discharge to home, } \\
\text { whichever comes first }\end{array}$ & $\begin{array}{l}>28 \mathrm{~d} \text { but }<56 \mathrm{~d} \text { postnatal age or discharge to home, } \\
\text { whichever comes first }\end{array}$ \\
\hline Treatment with oxygen $>\mathbf{2 1 \%}$ for at least $\mathbf{2 8} \mathrm{d}$ plus \\
\hline Mild BPD & $\begin{array}{l}\text { Breathing room air at } 36 \mathrm{wk} \text { ' PMA or discharge, } \\
\text { whichever comes first }\end{array}$ & $\begin{array}{l}\text { Breathing room air by 56 d postnatal age or discharge, } \\
\text { whichever comes first }\end{array}$ \\
\hline Moderate BPD & $\begin{array}{l}\text { Need for }<30 \% \text { at } \mathbf{3 6} \text { wk' PMA or discharge, } \\
\text { whichever comes first }\end{array}$ & $\begin{array}{l}\text { Need for }<30 \% \text { at 56 d postnatal age or discharge, } \\
\text { whichever comes first }\end{array}$ \\
\hline Severe BPD & $\begin{array}{l}\text { Need for } \geq 30 \% \text { oxygen and/or positive pressure } \\
\text { (PPV or NCPAP) at 36 wk' PMA or discharge, } \\
\text { whichever comes first }\end{array}$ & $\begin{array}{l}\text { Need for } \geq 30 \% \text { oxygen and/or positive pressure } \\
\text { (PPV or NCPAP) at 56 d postnatal age or discharge, } \\
\text { whichever comes first }\end{array}$ \\
\hline
\end{tabular}

Abbreviations: BPD, bronchopulmonary dysplasia; NCPAP, nasal continuous positive airway pressure; NIH, National Institutes of Health; PMA, postmenstrual age; PPV, positive pressure ventilation.

Source: Adapted from Jobe and Bancalari. ${ }^{4}$

time of assessment (physiologic definition) or to simplify the use in large populations. This has resulted in the use of different definitions of BPD not only across trials but also in clinical practice, thereby making meaningful comparisons of outcomes very difficult. ${ }^{5}$ - Fig. 1 shows the incidence of BPD in a group of extremely low birth weight infants using some of the definitions that have been suggested in different publications.

\section{Pitfalls of the Current Definition of Bronchopulmonary Dysplasia}

BPD is one of the few diseases arbitrarily defined by its treatment rather than by its pathophysiology or clinical picture. This potentially has resulted in lumping together of infants with diverse respiratory pathologies and subsequent long-term outcomes. This limitation is reflected in an incon- sistent association of diagnosis of BPD with long-term respiratory outcomes across different studies. This has brought to question the value of BPD diagnosis as an outcome measure for various antenatal and postnatal treatment strategies.

Another important element in the definition of BPD is to find an optimum timing of assessment. Though a continuous assessment of respiratory support over a period of time, such as area under the curve, is likely to more accurately reflect the severity of lung disease, it would be time demanding and more difficult to achieve in research or clinical settings. As reported by Isayama et al, the predictive accuracy of the definition of BPD for later respiratory outcomes progressively improves by delaying the time of assessment from 34 to 44weeks'PMA. ${ }^{6}$ However, this improvement in accuracy comes at the cost of decreasing the number of infants who can be classified prior to transfer or discharge from the hospital.

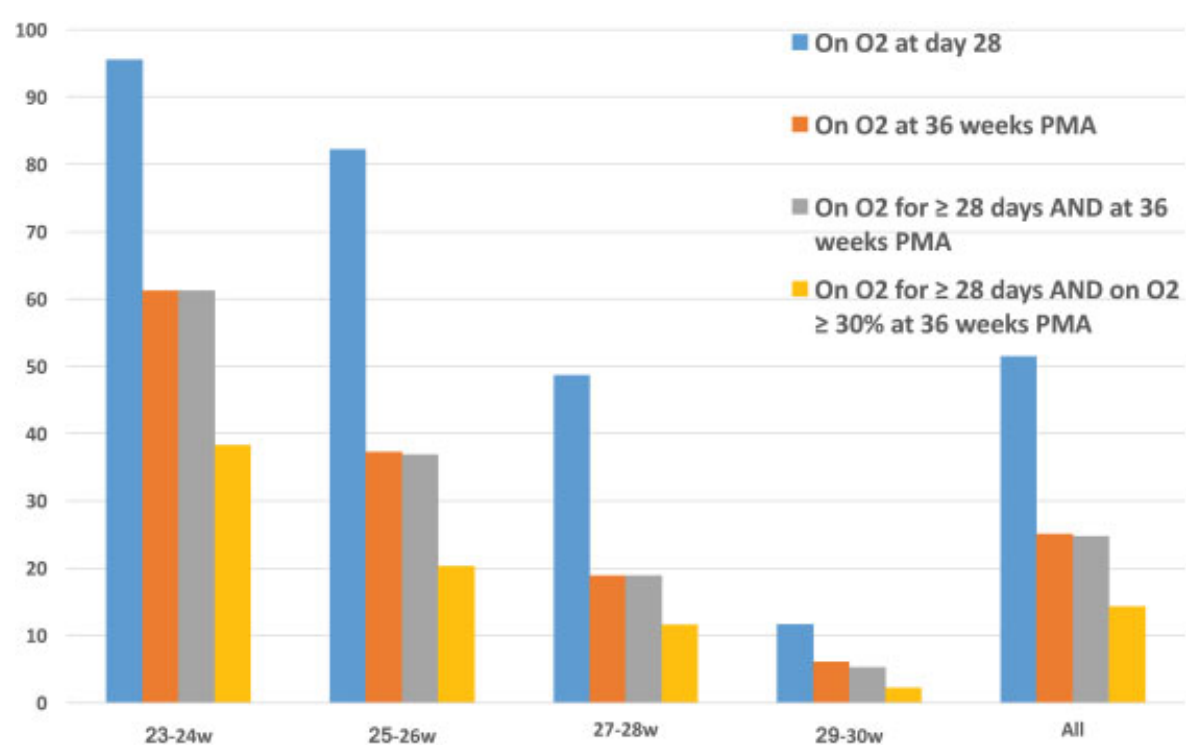

Fig. 1 Incidence of bronchopulmonary dysplasia (BPD) by diagnostic criteria across gestational ages (GAs). The figure shows the incidence of BPD according to different diagnostic criteria of BPD across different GAs. A large proportion of infants in lower GA groups need oxygen at day 28. Nearly two-thirds of those infants remain oxygen dependent at 36 weeks'postmenstrual age (PMA) and approximately one-third of them need $\geq 30 \%$ oxygen. Data are shown from 1,539 infants of 23 to 30 weeks' GA admitted to the neonatal intensive care unit of Holtz Children's Hospital of the Jackson Health System/University of Miami during the years 2005 to 2015 who were alive at 36 weeks' PMA. 
One of the significant limitations of current definitions of BPD is the inability to classify infants dying with severe respiratory failure before 36 weeks' PMA. These infants reflect a significant subset of extremely preterm infants who are likely to be the most at risk for developing severe BPD if they survived. ${ }^{7}$ One of the problems of including these infants in any classification of BPD is that it is very difficult to consistently differentiate between mortality from severe respiratory failure and other causes of death.

Some of the changes in clinical practice over the last decade have posed additional challenges to the definition of BPD. The increasing use of high-flow nasal cannula oxygen therapy has resulted in a new respiratory support strategy that combines oxygen supplementation with a variable degree of positive end-expiratory pressure. The classification of these infants on the basis of oxygen concentration alone, not including the positive pressure that they receive, may result in inaccurate estimation of lung disease severity. On the other extreme are the infants who receive $100 \%$ oxygen at very low nasal cannula flows. The classification of these infants on the basis of nasal cannula oxygen is currently a challenge as there are very limited data on the effect of different nasal cannula flows on effective fraction of inspired oxygen and airway pressure. ${ }^{8}$

Another group of infants who are difficult to classify as BPD are those requiring positive-pressure ventilation for abnormal respiratory control or severe airway abnormalities with no or minimal parenchymal lung disease. These infants should not be classified as BPD, but their proper classification would require some degree of clinical, radiographic, and/or functional assessment of parenchymal lung disease, all of which are subject to interpretation, have low specificity, and are difficult to apply in a large clinical population.

\section{The Way Forward}

The definition of BPD has different meanings for different stakeholders. While for an epidemiologist, a definition should accurately reflect the disease process and should be easy to use across neonatal units over time, for clinicians and researchers involved in developing preventive or treatment strategies, a definition with some correlation with long-term outcome is important. On the other hand, for a parent of an infant admitted to a neonatal intensive care unit, survival and the effect on long-term respiratory health is paramount. A key challenge for an ideal definition of BPD is to devise objective diagnostic criteria to appropriately categorize infants with different severity of lung disease while excluding infants with nonpulmonary causes of respiratory insufficiency. In addition, these criteria will need to be simple to use and require no or minimal extra effort by caregivers to ensure consistent use in large populations in different clinical settings.

One of the key issues with the diagnostic criteria of BPD has been the prediction of later respiratory course. This good intention is fraught with a risk of coming short on expectations since unlike a dichotomous definition of presence or absence of BPD, lung development and injury is a continuum from normal to severe dysfunction. ${ }^{9}$ In addition, lung devel- opment, additional injury, and the repair process continue well beyond the time of diagnosis of BPD and can be influenced by various factors after discharge from the hospital. With increasing survival of more immature infants, many of them meet the criteria of oxygen use at 36 weeks and are diagnosed as BPD, but a large proportion of them have only mild or minimal problems with long-term respiratory health. As expected, the diagnosis of severe BPD is the one more consistently associated with worse long-term respiratory outcomes. ${ }^{10}$ It is these infants with severe lung injury who must be properly classified so that they can be the primary target for prevention or treatment strategies that should improve their long-term outcome.

Recently, there has been increasing realization of the inadequacies of current definitions of BPD, and a concerted effort is being made to refine the diagnostic criteria and reflect current clinical practices more accurately. ${ }^{11}$ These new criteria will need to include infants dying of severe respiratory failure prior to 36weeks' PMA, categorize infants on different flows of nasal cannula oxygen, and provide specific guidance for including only infants with significant parenchymal lung disease. The National Institute of Child Health and Development recently held a workshop focused on BPD and its definition, and a report will be published in the near future. Longterm biomarkers for evaluating lung injury, ${ }^{12}$ imaging studies such as functional magnetic resonance imaging (MRI) to evaluate lung structure, ${ }^{13}$ and innovative ways to reliably assess pulmonary function will be needed to move toward a new classification of BPD that is more based on pathophysiology and objective lung function evaluation.

In conclusion, it is unlikely that a new definition of BPD will fulfill the requirements of all the stakeholders, but a consensus definition, which is relatively simple to use, encompasses current clinical practices, and provides a reasonable prediction of respiratory outcomes, is attainable and will improve the care of these infants.

\section{References}

1 Northway WH Jr, Rosan RC, Porter DY. Pulmonary disease following respirator therapy of hyaline-membrane disease. Bronchopulmonary dysplasia. N Engl J Med 1967;276(07):357-368

2 Shennan AT, Dunn MS, Ohlsson A, Lennox K, Hoskins EM. Abnormal pulmonary outcomes in premature infants: prediction from oxygen requirement in the neonatal period. Pediatrics 1988;82 (04):527-532

3 Tooley WH. Epidemiology of bronchopulmonary dysplasia.J Pediatr 1979;95(5 Pt 2):851-858

4 Jobe AH, Bancalari E. Bronchopulmonary dysplasia. Am J Respir Crit Care Med 2001;163(07):1723-1729

5 Hines D, Modi N, Lee SK, et al; International Network for Evaluating Outcomes (iNeo) of Neonates. Scoping review shows wide variation in the definitions of bronchopulmonary dysplasia in preterm infants and calls for a consensus. Acta Paediatr 2017;106 (03):366-374

6 Isayama T, Lee SK, Yang J, et al; Canadian Neonatal Network and Canadian Neonatal Follow-Up Network Investigators. Revisiting the definition of bronchopulmonary dysplasia: effect of changing panoply of respiratory support for preterm neonates. JAMA Pediatr 2017;171(03):271-279 
7 Poindexter BB, Feng R, Schmidt B, et al; Prematurity and Respiratory Outcomes Program. Comparisons and limitations of current definitions of bronchopulmonary dysplasia for the prematurity and respiratory outcomes program. Ann Am Thorac Soc 2015;12(12):1822-1830

8 Walsh M, Engle W, Laptook A, et al; National Institute of Child Health and Human Development Neonatal Research Network. Oxygen delivery through nasal cannulae to preterm infants: can practice be improved? Pediatrics 2005;116(04):857-861

9 Saarenpää HK, Tikanmäki M, Sipola-Leppänen M, et al. Lung function in very low birth weight adults. Pediatrics 2015;136(04): $642-650$
10 Ehrenkranz RA, Walsh MC, Vohr BR, et al; National Institutes of Child Health and Human Development Neonatal Research Network. Validation of the National Institutes of Health consensus definition of bronchopulmonary dysplasia. Pediatrics 2005;116(06):1353-1360

11 Jobe AH, Steinhorn R. Can we define bronchopulmonary dysplasia? J Pediatr 2017;188:19-23

12 Lal CV, Ambalavanan N. Cellular and humoral biomarkers of bronchopulmonary dysplasia. Early Hum Dev 2017;105:35-39

13 Walkup LL, Tkach JA, Higano NS, et al. Quantitative magnetic resonance imaging of bronchopulmonary dysplasia in the neonatal intensive care unit environment. Am J Respir Crit Care Med 2015;192(10):1215-1222 